\title{
Oral Lipid-Lowering Treatments Beyond Statins: Too Old and Outdated or Still Useful?
}

\author{
Klaus G. Parhofer ${ }^{1}$ \\ Accepted: 5 August 2021 / Published online: 14 October 2021 \\ (c) The Author(s) 2021
}

\begin{abstract}
Purpose of Review For many years, the lipid-lowering armamentarium consisted of statins and/or ezetimibe and/or bile acid sequestrants and/or fibrates. Now, with the availability of new drugs mostly injectables, the field has changed and the role of oral non-statin drugs (including bempedoic acid) must be reevaluated.

Recent Findings Ezetimibe remains a very important combination partner for statins with continuously increasing treatment numbers. Bempedoic acid is another interesting combination partner for statin/ezetimibe or ezetimibe alone but lacks in contrast to ezetimibe evidence from outcome trials. The role of fibrates is less clear as they have shown disappointing results in outcome trials but may still be used in selected, high-risk patients with combined dyslipidemia. Bile acid sequestrants are now rarely used as there are stronger, better tolerable ways to lower LDL-cholesterol.

Summary With the introduction of new injectable lipid-lowering drugs, some oral drugs such as ezetimibe and bempedoic acid still have an important spot in our treatment algorithm others such as fibrates have a less clear role while again others are now rarely used.
\end{abstract}

Keywords Ezetimibe $\cdot$ Bempedoic acid $\cdot$ Colesevelam $\cdot$ Fibrates

\section{Introduction}

The knowledge that lipids play an important role in atherogenesis and that lipid lowering translates into risk reduction for cardiovascular events has promoted the development of a number of new lipid-lowering drugs which can dramatically change the level of atherogenic lipoproteins. This is particularly true for PCSK9 antibodies such as alirocumab and evolocumab or PCSK9 inhibitors based on siRNA technology such as inclisiran but is also true for drugs used in rare conditions such as volanesorsen in familial chylomicronemia syndrome. In addition, a number of new drugs addressing ANGPTL-3, apo(a) or apoCIII, or other components important for plasma lipid levels are being developed. Most of these new drugs will be injectables as they are either antibodies or oligonucleotides.

This article is part of the Topical Collection on Nonstatin Drugs

Klaus G. Parhofer

klaus.parhofer@med.uni-muenchen.de

1 Medizinische Klinik Und Poliklinik IV, LMU Klinikum, Marchioninistr. 15, 81377 Munich, Germany
The availability of such potent drugs to modify lipid levels raises the important question of whether and to what extent oral lipid-lowering drugs should be used. Statins are the basic lipidlowering therapy and will probably remain so for many years as they are cheap, widely available, and supported by numerous convincing outcome trials. In addition, the safety profile of statins is extremely good. However, the positioning of other oral lipid-lowering drugs (Table 1) often used in combination with statins may be less clear. It is questionable whether they can keep their present role considering that the newer injectable drugs mentioned above are usually also used in combination with statins and show a convincing efficacy and safety profile.

The purpose of this review is to pigeonhole the role of these oral drugs in the current environment of new (expensive) injectable lipid-lowering drugs. This review will discuss ezetimibe, bempedoic acid, fibrates, and bile sequestrants.

\section{Ezetimibe}

Ezetimibe is a cholesterol absorption inhibitor, which blocks the Niemann-Pick C1 like 1 protein (NPC1L1) in the gastrointestinal tract (as well as in hepatocytes). 


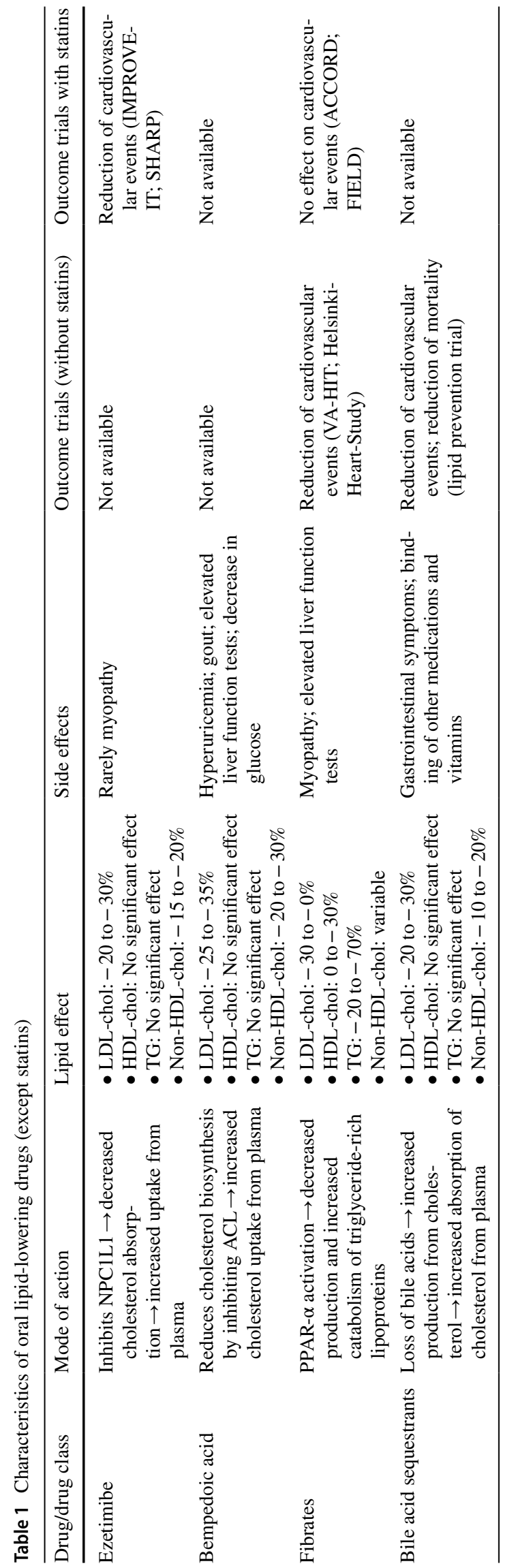

Blocking intestinal absorption of cholesterol results in an upregulation of LDL-receptors which consequently reduces plasma LDL-cholesterol levels [1]. Ezetimibe is on the market for almost 20 years.

In combination with statins, ezetimibe can decrease LDL-cholesterol on average by $20-25 \%$ although the effect may vary depending on whether the treated subject is a high or low absorber [2]. Ezetimibe is very well tolerated with almost no side effects of its own. Despite this relevant LDL-cholesterol reduction and very benign side-effect profile, ezetimibe was used reluctantly until the publication of the outcome trial in 2015. Since then, the use of ezetimibe alone or in combination with statins has increased and is further increasing. Most guidelines and recommendations now favor statin ezetimibe combination if statin therapy by itself is not sufficient to reach treatment targets [3]. Most recommendations propagate a sequential approach with using statin monotherapy first and then adding ezetimibe if necessary. However, in some patients with severely elevated LDL-cholesterol (large distance to goal), primary combination therapy may be an option. This may especially be the case when follow-up visits are difficult to implement. Ezetimibe is also used in many patients with statin intolerance as ezetimibe itself has basically no side effects [3, 4].

Ezetimibe was tested in a large outcome trial (IMPROVE-IT Study) in which the combination of simvastatin with ezetimibe was compared to simvastatin monotherapy in 18,144 patients with acute coronary syndrome [5•]. The reduction of LDL-cholesterol from $69.5 \mathrm{mg} / \mathrm{dl}$ (simvastatin monotherapy) to $53.7 \mathrm{mg} / \mathrm{dl}$ (simvastatin/ ezetimibe group) resulted in a significant $6.4 \%$ risk reduction $(0.89-0.99 ; p=0.016)$. Further subgroup analysis indicates that particularly patients at high risk benefited from this additional decrease in LDL-cholesterol [6]. The IMPROVE-IT trial can be considered as a milestone study as it was the first study to show that LDL-cholesterol levels around $50 \mathrm{mg} / \mathrm{dl}$ are more effective than those around $70 \mathrm{mg} / \mathrm{dl}$ with respect to risk reduction. It was also the first study to show that LDL lowering in combination with statins translates into benefit and finally the study gave ultimate proof for the safety of ezetimibe. In a further outcome trial, the combination of simvastatin and ezetimibe was tested in patients with renal insufficiency, again showing significant risk reduction [7]. Although the study lacks a statin monotherapy arm, it is interesting, as previous studies using atorvastatin or rosuvastatin did not show benefit in patients with end-stage renal disease.

Furthermore, newer data indicate that NPC1L1 may also play a role in non-alcoholic fatty liver disease (NAFLD) and non-alcoholic steatohepatitis (NASH) [8]. In fact, a recent meta-analysis shows that ezetimibe can attenuate serum liver enzymes and hepatic steatosis and ballooning in fatty liver disease [9]. However, larger studies including randomized 
controlled trials are necessary to determine ezetimibe's clinical role in the management of fatty liver disease.

In conclusion, ezetimibe is an effective and safe drug that can decrease LDL-cholesterol in addition to statin therapy and this additional reduction translates into clinical benefit (Table 2). It can be assumed that ezetimibe will remain the primary partner for statins, if statins alone are not sufficient to reach the LDL-cholesterol goal. Its role in fatty liver disease needs to be determined.

\section{Bempedoic Acid}

Bempedoic acid is a new oral lipid-lowering drug which has been introduced to the European market in November 2020. Similar to statins, bempedoic acid reduces cholesterol biosynthesis [10]. While statins inhibit HMG-CoA-reductase, bempedoic acid inhibits ATP-citrat-lyase (ACL) [11]. In order to inhibit ACL, bempedoic acid has to be activated by long-chain acyl-CoA-synthetase-1. This activation can only take place in hepatocytes. Therefore, muscle-related side effects seen with statin therapy in approximately $10 \%$ of patients should not occur when patients are treated with bempedoic acid. This is supported by data from phase 2 and 3 studies, in which it was shown that muscle-related side

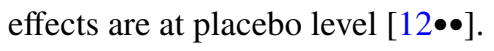

Although bempedoic acid inhibits the same pathway as statins, bempedoic acid can decrease LDL-cholesterol even

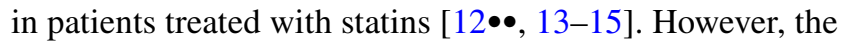
effect on LDL-cholesterol is less pronounced when used with high-dose statins. In monotherapy, LDL-cholesterol can be decreased by approximately $20 \%$. In combination with ezetimibe, reductions of up to $35 \%$ can be observed.

Bempedoic acid may increase the dose of statins; therefore, the combination with high-dose simvastatin should be avoided [16]. Furthermore, bempedoic acid can increase uric acids levels which in predisposed patients may lead to hyperuricemia or gout. In phase 2 and phase 3 studies, increases in uric acid levels are observed in approximately $2 \%$ of patients, while acute gout occurred in $1.4 \%$ (compared to $0.4 \%$ on placebo). The risk of gout is considerably higher in patients with a history of gout ( $11 \%$ vs. $2.9 \%$ on placebo). Other rare adverse events relate to a small decrease in hemoglobin levels, the mechanism behind this is unclear. Finally, the use of bempedoic acid is associated with decrease in glucose levels (in contrast to statins) and also significantly decreases CRP (similar to statins).

Currently, no outcome data are available but it is expected that the CLEAR outcome study which examines the effect of bempedoic acid compared to placebo in statin-intolerant patients ( $>14,000$ patients, approximately 4.75 years observation period) will be presented in 2023. Genetic data strongly indicate that LDL-cholesterol reduction achieved with bempedoic acid will result in risk reduction [17].

Currently, bempedoic acid is mostly used in 2 clinical settings [16]. Primarily in patients with statin intolerance

Table 2 Use of oral lipid-lowering drugs (except statins) in clinical practice

\begin{tabular}{|c|c|c|}
\hline Drug/drug class & Clinical use & Predicted future role \\
\hline Ezetimibe & $\begin{array}{l}\text { - In combination with statins to further decrease LDL-cholesterol } \\
\text { - Together with low-dose statins (in statin intolerance) } \\
\text { - As monotherapy or in combination with other lipid-lowering drugs } \\
\text { (in patients with statin intolerance) }\end{array}$ & Use will probably increase \\
\hline Bempedoic acid & $\begin{array}{l}\text { - In combination with low-dose statins and/or ezetimibe (in statin } \\
\text { intolerance) } \\
\text { - In patients not reaching LDL-goal on moderate or high-dose statin } \\
\text { plus ezetimibe } \\
\text { - In combination with other lipid-lowering drugs (in statin intolerance) }\end{array}$ & Future role will largely depend on outcome trial \\
\hline Fibrates & $\begin{array}{l}\text { - In isolated hypertriglyceridemia to reduce risk for pancreatitis } \\
\text { - In combination with statins (selected patients) to reduce cardiovascu- } \\
\text { lar risk in patients with combined dyslipidemia } \\
\text { - To decrease LDL-cholesterol, if statins, ezetimibe, bempedoic acid, } \\
\text { and/or bile acid sequestrants are not tolerated or not available }\end{array}$ & $\begin{array}{l}\text { uSe will probably decrease, except if pemafi- } \\
\text { brate outcome trial is strongly positive }\end{array}$ \\
\hline Bile acid sequestrants & $\begin{array}{l}\text { - In LDL-hypercholesterolemia if other drugs are not tolerated or not } \\
\text { available } \\
\text { - As add on to statins and ezetimibe in patients with LDL-hypercholes- } \\
\text { terolemia not qualifying for PCSK9-inhibition } \\
\text { - In LDL-hypercholesterolemia and contraindication to systemic drugs } \\
\text { (pregnancy; children) } \\
\text { - CAVE: May aggravate hypertriglyceridemia in hypertriglyceridemic } \\
\text { subjects }\end{array}$ & Use will probably further decrease \\
\hline
\end{tabular}


in whom it can be assumed that a decrease LDL-cholesterol by approximately $30-40 \%$ will be sufficient to reach the treatment target. In that situation, bempedoic acid can be used together with different doses of statins and ezetimibe. Furthermore, bempedoic acid may also be used in situations where patients at high or very high risk do not reach the treatment target despite high-dose statin therapy in combination with ezetimibe. However, this approach is usually only successful if the distance to treatment target is not "too far."

In summary, bempedoic acid is an interesting new lipidlowering drug, which is primarily used in statin-intolerant subjects but may also play a role in patients not reaching LDL-cholesterol target despite maximal statin end/or ezetimibe and/or PCSK9-based therapy (Table 2). The future positioning of bempedoic acid will largely be determined by the results of the outcome trial.

\section{Fibrates}

Fibrates bind and activate PPAR- $\alpha$ [18]. Although the exact mechanism is not clear, the fibrate-induced activation of PPAR- $\alpha$ results in a decrease in apo CIII, an activation of lipoprotein lipase, and apo AV but also decreases the activity of diacylglycerolacyltransferase [19]. At the same time, apo $\mathrm{AI}$ and apo AII levels are increased. Overall, this results in a significant decrease of triglyceride levels (up to minus 70\%) and an increase in HDL-cholesterol (up to plus 30\%). The effect on LDL-cholesterol is variable and probably depends on the underlying dyslipidemia. While the primary action of fibrates seems to be the reduction of triglyceride levels, it is unclear whether the changes observed in HDL-metabolism are the result of PPAR- $\alpha$ activation or are just secondary to the triglyceride reduction.

In contrast to statin therapy, the effect of fibrate therapy is less predictable. This most likely represents the fact that there is a wide range of potential causes for hypertriglyceridemia (usually a combination of genetic predisposition and lifestyle factors). It should be noted that triglyceride reduction with fibrates is minimal in the most severe cases of hypertriglyceridemia such as familial chylomicronemia syndrome [20].

In monotherapy fibrates (usually gemfibrocil) can reduce the risk for atherosclerotic disease, but in combination with statins (used fibrate fenofibrate), no additional benefit was observed [21-24]. However, subgroup analyses of these outcome trials indicate that such combination therapy may reduce the risk in patients with hypertriglyceridemia and low HDL-cholesterol [25•]. It is currently unclear whether the failure to show risk reduction in combination trials relates to true failure or to methodological aspects related to study design. Pemafibrate, a new potent fibrate, is currently being tested in a large outcome trial and results will probably be reported in 2021 or 2022 [26, 27].

While the currently available outcome trials are disappointing with respect to cardiovascular risk reduction, they surprisingly showed a significant effect of fenofibrate on microvascular complications in diabetic patients [28]. The effect was particularly strong for retinopathy in the FIELD trial and resulted in the approval of fenofibrate for treatment of diabetic retinopathy in some countries.

From a clinical perspective, fibrates are used in patients with hypertriglyceridemia either as monotherapy in patients with isolated hypertriglyceridemia and low cardiovascular risk or in combination with LDL-cholesterol-lowering drugs if LDL-cholesterol is reached and risk is perceived as high or very high. No clear-cut recommendations when to use fibrates are currently available, which is mostly due to the lack of convincing outcome trials (as outlined above) [3, 29].

In conclusion, fibrates still play a role in patients with combined dyslipidemia or isolated hypertriglyceridemia but due to the lack of convincing outcome trials, the decision should be individualized (Table 2). In addition, it should be noted that soon, eicosapentainic acid ethylester will be available which may further decrease the role of fibrates (especially if the pemafibrate trial is negative).

\section{Bile Acid Sequestrants}

Bile acid sequestrants such as colesevelam, colestipol, or colestyramine bind bile acids in the gut and thereby prevent the reabsorption of bile acids. This results in the loss of bile acids from the enterohepatic circulation. This depletion of bile acids leads to an increased production of bile acids for which cholesterol is required $[30,31]$. Therefore, hepatic uptake of cholesterol from the circulation is increased (as well as biosynthesis). A daily dose of 8-24 g of cholestyramine and $3.75 \mathrm{~g}$ of colesevelam must be used to induce significant decreases of LDL-cholesterol. Interestingly, colesevelam has consistently shown glucose-lowering effects, which is particularly noteworthy as statins increase glucose levels [32]. Despite this, its role in diabetes treatment needs to be determined.

Typically, reductions in LDL-cholesterol of $20-30 \%$ can be observed. Importantly, triglyceride levels may increase during bile acid sequestrants therapy because the production of bile acids and VLDL is co-regulated [33]. Despite this significant effect on LDL-cholesterol, these drugs are rarely used in current practice because of the high rate of side effects. These include gastrointestinal symptoms, most commonly flatulence, constipation, dyspepsia, and nausea. Furthermore, the absorption of fat-soluble vitamins can be reduced. As bile acid sequestrants can also bind to many commonly prescribed drugs, they must be administered 
either $4 \mathrm{~h}$ before or $1 \mathrm{~h}$ after other drugs. Compared to colestyramine and colestipol, colesevelam is better tolerated and has fewer interactions with other medications. On the other hand, bile acid sequestrants are not systemically absorbed and can therefore be used in children and pregnant women.

Bile acid sequestrants have shown benefit in large outcome trials (before the availability of modern treatment options) [34]. No outcome data are available in combination with statin therapy.

Currently, bile acid sequestrants are only used as thirdline options for decreasing LDL-cholesterol and are only used in selected patients and situations (Table 2). It can be assumed that with newer and more potent drugs available to decrease LDL-cholesterol, the role will be further reduced.

\section{Conclusion}

Although a number of very potent, very effective, and safe lipid-lowering injectables are being developed or introduced to the market, there remains space for oral lipid-lowering drugs besides statins. Some of them, such as ezetimibe and bempedoic acid, will probably further grow, while the future of others, such as fibrates and bile acid sequestrants, is less clear.

Funding Open Access funding enabled and organized by Projekt DEAL.

\section{Declarations}

Conflict of Interest Dr. Parhofer reports personal fees from Akcea, Amarin, Amgen, Daiichi-Sankyo, Ionis, MSD, Novartis, and Sanofi, outside the submitted work.

Human and Animal Rights and Informed Consent This article does not contain any studies with human or animal subjects performed by any of the authors.

Open Access This article is licensed under a Creative Commons Attribution 4.0 International License, which permits use, sharing, adaptation, distribution and reproduction in any medium or format, as long as you give appropriate credit to the original author(s) and the source, provide a link to the Creative Commons licence, and indicate if changes were made. The images or other third party material in this article are included in the article's Creative Commons licence, unless indicated otherwise in a credit line to the material. If material is not included in the article's Creative Commons licence and your intended use is not permitted by statutory regulation or exceeds the permitted use, you will need to obtain permission directly from the copyright holder. To view a copy of this licence, visit http://creativecommons.org/licenses/by/4.0/.

\section{References}

Papers of particular interest, published recently, have been highlighted as:

- Of importance

$\bullet$ Of major importance

1. Temel RE, Tang W, Ma Y, Rudel LL, Willingham MC, Ioannou YA, et al. Hepatic Niemann-Pick C1-like 1 regulates biliary cholesterol concentration and is a target of ezetimibe. J Clin Invest. 2007;117:1968-78.

2. Leiter LA, Betteridge DJ, Farnier M, Guyton JR, Lin J, Shah A, et al. Lipid-altering efficacy and safety profile of combination therapy with ezetimibe/statin vs. statin monotherapy in patients with and without diabetes: an analysis of pooled data from 27 clinical trials. Diabetes Obes Metab. 2011;13:615-28.

3. Mach F, Baigent C, Catapano AL, Koskinas KC, Casula M, Badimon L, et al. 2019 ESC/EAS Guidelines for the management of dyslipidaemias: lipid modification to reduce cardiovascular risk. Eur Heart J. 2020;41:111-88.

4. Stroes ES, Thompson PD, Corsini A, Vladutiu GD, Raal FJ, Ray $\mathrm{KK}$, et al. Statin-associated muscle symptoms: impact on statin therapy-European Atherosclerosis Society Consensus Panel Statement on Assessment. Aetiology and Management Eur Heart J. 2015;36:1012-22.

5. Cannon CP, Blazing MA, Giugliano RP, McCagg A, White JA, Theroux P, et al. Ezetimibe added to statin therapy after acute coronary syndromes. N Engl J Med. 2015;372:2387-97. First outcome trial that showed benefit for a lipid-lowering drug (ezetimibe) given in combination with a statin. Also confirmed the concept "the lower the better" and proved the safety of ezetimibe.

6. Bohula EA, Morrow DA, Giugliano RP, Blazing MA, He P, Park JG, et al. Atherothrombotic risk stratification and ezetimibe for secondary prevention. J Am Coll Cardiol. 2017;69:911-21.

7. Baigent C, Landray MJ, Reith C, Emberson J, Wheeler DC, Tomson C, et al. The effects of lowering LDL cholesterol with simvastatin plus ezetimibe in patients with chronic kidney disease (Study of Heart and Renal Protection): a randomised placebo-controlled trial. Lancet. 2011;377:2181-92.

8. Toyoda Y, Takada T, Umezawa M, Tomura F, Yamanashi Y, Takeda K, et al. Identification of hepatic NPC1L1 as an NAFLD risk factor evidenced by ezetimibe-mediated steatosis prevention and recovery. FASEB Bioadv. 2019;1:283-95.

9. Nakade Y, Murotani K, Inoue T, Kobayashi Y, Yamamoto T, Ishii N, et al. Ezetimibe for the treatment of non-alcoholic fatty liver disease: a meta-analysis. Hepatol Res. 2017;47:1417-28.

10. Masana Marin L, Plana Gil N. Bempedoic acid. Mechanism of action and pharmacokinetic and pharmacodynamic properties. Clin Investig Arterioscler. 2021;33 Suppl 1:53-7.

11. Pinkosky SL, Filippov S, Srivastava RA, Hanselman JC, Bradshaw CD, Hurley TR, et al. AMP-activated protein kinase and ATP-citrate lyase are two distinct molecular targets for ETC1002 , a novel small molecule regulator of lipid and carbohydrate metabolism. J Lipid Res. 2013;54:134-51.

12.•• Ray KK, Bays HE, Catapano AL, Lalwani ND, Bloedon LT, Sterling LR, et al. Safety and efficacy of bempedoic acid to reduce LDL cholesterol. N Engl J Med. 2019;380:1022-32. This study shows that bempedoic acid can decrease LDLcholesterol in patients on low, moderate and high dose statin therapy. It also describes the kind and frequencies of side effects.

13. Ballantyne CM, Laufs U, Ray KK, Leiter LA, Bays HE, Goldberg AC, et al. Bempedoic acid plus ezetimibe fixed-dose 
combination in patients with hypercholesterolemia and high CVD risk treated with maximally tolerated statin therapy. Eur J Prev Cardiol. 2020;27:593-603.

14. Goldberg AC, Leiter LA, Stroes ESG, Baum SJ, Hanselman JC, Bloedon LT, et al. Effect of bempedoic acid vs placebo added to maximally tolerated statins on low-density lipoprotein cholesterol in patients at high risk for cardiovascular disease: the CLEAR wisdom randomized clinical trial. JAMA. 2019;322:1780-8.

15. Rubino J, MacDougall DE, Sterling LR, Hanselman JC, Nicholls SJ. Combination of bempedoic acid, ezetimibe, and atorvastatin in patients with hypercholesterolemia: a randomized clinical trial. Atherosclerosis. 2021;320:122-8.

16. Ballantyne CM, Bays H, Catapano AL, Goldberg A, Ray KK, Saseen JJ. Role of bempedoic acid in clinical practice. Cardiovasc Drugs Ther. 2021.

17. Ference BA, Ray KK, Catapano AL, Ference TB, Burgess S, Neff DR, et al. Mendelian randomization study of ACLY and cardiovascular disease. N Engl J Med. 2019;380:1033-42.

18. Schoonjans K, Peinado-Onsurbe J, Lefebvre AM, Heyman RA, Briggs M, Deeb S, et al. PPARalpha and PPARgamma activators direct a distinct tissue-specific transcriptional response via a PPRE in the lipoprotein lipase gene. EMBO J. 1996;15:5336-48.

19. Mehlum A, Staels B, Duverger N, Tailleux A, Castro G, Fievet $\mathrm{C}$, et al. Tissue-specific expression of the human gene for lecithin: cholesterol acyltransferase in transgenic mice alters blood lipids, lipoproteins and lipases towards a less atherogenic profile. Eur J Biochem. 1995;230:567-75.

20. Stroes E, Moulin P, Parhofer KG, Rebours V, Lohr JM, Averna M. Diagnostic algorithm for familial chylomicronemia syndrome. Atheroscler Suppl. 2017;23:1-7.

21 Frick MH, Elo O, Haapa K, Heinonen OP, Heinsalmi P, Helo P, et al. Helsinki Heart Study: primary-prevention trial with gemfibrozil in middle-aged men with dyslipidemia. Safety of treatment, changes in risk factors, and incidence of coronary heart disease. N Engl J Med. 1987;317:1237-45.

22. Ginsberg HN, Elam MB, Lovato LC, Crouse JR 3rd, Leiter LA, Linz $P$, et al. Effects of combination lipid therapy in type 2 diabetes mellitus. N Engl J Med. 2010;362:1563-74.

23. Rubins HB, Robins SJ, Collins D, Fye CL, Anderson JW, Elam $\mathrm{MB}$, et al. Gemfibrozil for the secondary prevention of coronary heart disease in men with low levels of high-density lipoprotein cholesterol. Veterans Affairs High-Density Lipoprotein Cholesterol Intervention Trial Study Group. N Engl J Med. $1999 ; 341: 410-8$.
24. A co-operative trial in the primary prevention of ischaemic heart disease using clofibrate. Report from the Committee of Principal Investigators. Br Heart J. 1978;40:1069-118.

25. Jun M, Foote C, Lv J, Neal B, Patel A, Nicholls SJ, et al. Effects of fibrates on cardiovascular outcomes: a systematic review and meta-analysis. Lancet. 2010;375:1875-84. Summarizes the current evidence concerning the effect of fibrates on cardiovascular outcomes. Highlights which subgoups may benefit from fibrate therapy.

26. Araki E, Yamashita S, Arai H, Yokote K, Satoh J, Inoguchi $\mathrm{T}$, et al. Effects of pemafibrate, a novel selective PPARalpha modulator, on lipid and glucose metabolism in patients with type 2 diabetes and hypertriglyceridemia: a randomized, double-blind, placebo-controlled, phase 3 trial. Diabetes Care. 2018;41:538-46.

27. Pradhan AD, Paynter NP, Everett BM, Glynn RJ, Amarenco $\mathrm{P}$, Elam M, et al. Rationale and design of the pemafibrate to reduce cardiovascular outcomes by reducing triglycerides in patients with diabetes (PROMINENT) study. Am Heart J. 2018;206:80-93.

28 Simo R, Hernandez C. Prevention and treatment of diabetic retinopathy: evidence from large, randomized trials. The emerging role of fenofibrate. Rev Recent Clin Trials. 2012;7:71-80.

29. Laufs U, Parhofer KG, Ginsberg HN, Hegele RA. Clinical review on triglycerides. Eur Heart J. 2020;41:99-109c.

30. Ast M, Frishman WH. Bile acid sequestrants. J Clin Pharmacol. 1990;30:99-106.

31. Bays H, Dujovne C. Colesevelam HCl: a non-systemic lipidaltering drug. Expert Opin Pharmacother. 2003;4:779-90.

32 Ooi CP, Loke SC. Colesevelam for type 2 diabetes mellitus. Cochrane Database Syst Rev. 2012;12:CD009361.

33. Beil U, Crouse JR, Einarsson K, Grundy SM. Effects of interruption of the enterohepatic circulation of bile acids on the transport of very low density-lipoprotein triglycerides. Metabolism. 1982;31:438-44.

34. Lipid Research Clinics Program. The Lipid Research Clinics Coronary Primary Prevention Trial results. I. Reduction in incidence of coronary heart disease. JAMA. 1984;251:351-64.

Publisher's Note Springer Nature remains neutral with regard to jurisdictional claims in published maps and institutional affiliations. 